\title{
Optimization models of a two-component nuclear energy system with thermal and fast reactors in a closed nuclear fuel cycle*
}

\author{
Andrey A. Andrianov ${ }^{1}$, Ilya S. Kuptsov ${ }^{1}$, Tatyana A. Osipova ${ }^{1}$, Olga N. Andrianova ${ }^{2}$, \\ Tatyana V. Utyanskaya ${ }^{3}$ \\ 1 Obninsk Institute of Nuclear Power Engineering, National Research Nuclear University "MEPhI", 1 Studgorodok, Obninsk, Kaluga reg., \\ 249040 Russia \\ 2 JSC "SSC RF-IPPE n.a. A.I. Leypunsky”, 1, Bondarenko sq., Obninsk, Kaluga reg., 249033 Russia \\ 3 JSC "Engineering Center of Nuclear Containers", Bldg 1, Marshala Biryuzova st., Moscow, 123298 Russia
}

Corresponding author: Andrey A. Andrianov (andreyandrianov@yandex.ru)

Academic editor: G. Tikhomirov • Received 26 November 2018 • Accepted 20 January 2019 • Published 20 March 2019

Citation: Andrianov AA, Kuptsov IS, Osipova TA, Andrianova ON, Utyanskaya TV (2019) Optimization models of a two-component nuclear energy system with thermal and fast reactors in a closed nuclear fuel cycle. Nuclear Energy and Technology 5(1): 39-45. https://doi.org/10.3897/nucet.5.33981

\begin{abstract}
The article presents a description and some illustrative results of the application of two optimization models for a two-component nuclear energy system consisting of thermal and fast reactors in a closed nuclear fuel cycle. These models correspond to two possible options of developing Russian nuclear energy system, which are discussed in the expert community: (1) thermal and fast reactors utilizing uranium and mixed oxide fuel, (2) thermal reactors utilizing uranium oxide fuel and fast reactors utilizing mixed nitride uranium-plutonium fuel. The optimization models elaborated using the IAEA MESSAGE energy planning tool make it possible not only to optimize the nuclear energy system structure according to the economic criterion, taking into account resource and infrastructural constraints, but also to be used as a basis for developing multi-objective, stochastic and robust optimization models of a two-component nuclear energy system. These models were elaborated in full compliance with the recommendations of the IAEA's PESS and INPRO sections, regarding the specification of nuclear energy systems in MESSAGE. The study is based on publications of experts from NRC "Kurchatov Institute", JSC "SSC RF-IPPE”, ITCP "Proryv", JSC "NIKIET". The presented results demonstrate the characteristic structural features of a two-component nuclear energy system for conservative assumptions in order to illustrate the capabilities of the developed optimization models. Consideration is also given to the economic feasibility of a technologically diversified nuclear energy structure providing the possibility of forming on its base a robust system in the future. It has been demonstrated that given the current uncertainties in the costs of nuclear fuel cycle services and reactor technologies, it is impossible at the moment to make a reasonable conclusion regarding the greatest attractiveness of a particular option in terms of the economic performance.
\end{abstract}

\section{Keywords}

Thermal reactors; fast reactors; closed nuclear fuel cycle; UOX; MOX; MNUP; optimization; uncertainty; forecasting;

* Russian text published: Izvestiya vuzov. Yadernaya Energetika (ISSN 0204-3327), 2018, n. 3, pp. 100-112. 


\section{Introduction}

Currently, of all possible options for the national nuclear energy development, two alternatives are most actively discussed: they are denoted by the common term, i.e., two-component nuclear energy system (NES). It is assumed that such a system at different time horizons may contain in its composition both thermal and fast reactors coupled by a closed nuclear fuel cycle (NFC) (Ponomarev-Stepnoy 2016, Alekseev et al. 2011, Alekseev et al. 2012, Alekseev et al. 2017, Adamov 2001, Adamov et al. 2012, Adamov et al. 2017, Muravyev 2014).

One of the options (hereafter Option 1) assumes that the thermal and fast reactors will run on pellet oxide fuel; at the same time, thermal reactors can use both uranium oxide and mixed uranium-plutonium (MOX) fuels, and fast reactors with $\mathrm{BR}>1$ can operate on MOX fuel. It is also assumed that plutonium from spent nuclear fuel (SNF) of thermal reactors will be used as fuel for fast reactors, and plutonium from SNF (including the blanket) of fast reactors can also be used to produce MOX fuel for thermal reactors. This option involves centralized SNF reprocessing and fuel production (Ponomarev-Stepnoy 2016, Alekseev et al. 2011, Alekseev et al. 2012, Alekseev et al. 2017).

In the second option (hereinafter Option 2), thermal reactors will continue to use uranium oxide fuel as long as there are resources of natural uranium available at an affordable cost, and fast reactors (with or without fuel breeding) will use mixed nitride uranium-plutonium (MNUP) fuel, plutonium for which can be extracted from SNF of both thermal and fast reactors. The implementation of both centralized and on-site NFC is assumed to be possible under this option (Adamov 2001, Adamov et al. 2012, Adamov et al. 2017, Muravyev 2014).

Both of these options have their own characteristics, advantages and disadvantages as well as certain similarities and differences that are discussed in the above publications. However, there are currently no studies in which a comparative analysis of both concepts is carried out at the system level, on a unified methodological and calculation basis, proceeding from common scenario and model assumptions. This article is partly aimed at filling this gap, focusing on identifying the characteristic structural features of the above concepts using optimization models of a two-component NES with thermal and fast reactors in a closed NFC.

\section{Problem statement and initial data}

In analytical and foresight studies conducted to justify the development priorities of nuclear technologies, system models imitating the NES deployment are widely used. Corresponding software tools are divided into two categories: simulation and optimization instruments. For modelling the deployment of the two-component NES with thermal and fast reactors in a closed NFC, simulation models are currently widely used. The simulation models record all the main scenario assumptions (NES structure, SNF reprocessing schedule, etc.) and make it possible to estimate the material flows in the system, requirements for NFC services and products as well as relevant performance indicators. However, they do not indicate the trends of structural changes in the system to increase its performance.

The optimization tools compensate for this drawback of the simulation instruments, but remain a less popular tool for modeling the two-component NES deployment due to the large efforts required for the development of relevant optimization models, their testing, application and interpretation of results. It seems appropriate to extensively use the optimization models since they not only identify the most effective ways to improve the system performance in the framework of single-criterion and multi-objective paradigms but also allow highlighting the characteristic structural features of systems and evolution trends in view of uncertainties in the main scenario and technical-economic parameters. Due to this, it becomes possible to indicate directions that provide enhanced performance and robustness (stability in regard to potential changes in the technical, economic and scenario parameters) of NES structures. It is for these reasons that the present article is focused on the optimization models of a two-component NES with thermal and fast reactors in a closed NFC.

The MESSAGE energy planning software was used as the main calculation tool (developed by the International Institute for Applied Systems Analysis (IIASA), currently distributed and maintained by the IAEA, used as part of the software toolkit of the International Project on Innovative Reactors and Fuel Cycles (INPRO)) (Messner and Strubergger 1995, MESSAGE - Model). National experts, experts of the IAEA PESS and INPRO sections developed recommendations on the specification of the NES in MESSAGE, which meet state-of-the-art requirements in this subject-matter area (NG-T-5.2 2016, Andrianov et al. 2012). MESSAGE is widely used in national studies and is one of the benchmark software for verifying original codes (Andrianov et al. 2012, Andrianov 2012, Andrianov et al. 2016, Andrianov et al. 2010, Andrianov et al. 2017).

The aim of this study is to demonstrate the added value which can be provided by applying an optimization approach to the problem of searching and rationale for the most efficient structures of a two-component NES. For this purpose, it is necessary to identify the characteristic structural features of the models of the discussed two-component NES options, estimate the uncertainties 
in the levelized generation costs (LGC) ${ }^{1}$ and the NES structures caused by the uncertainty in the costs of NFC services and reactor technologies. The information base of the study consists of technical-economic data and scenario assumptions presented in the publications of experts from the NRC "Kurchatov Institute", JSC "SSC RF-IPPE", ITCP "Proryv", and JSC "NIKIET" (Ponomarev-Stepnoy 2016, Alekseev et al. 2011, Alekseev et al. 2012, Alekseev et al. 2017, Adamov 2001, Adamov et al. 2012, Adamov et al. 2017, Muravyev 2014).

\section{A brief description of optimization models}

The existing thermal reactors were combined into two groups: RBMK and VVER. In the future, the following reactor types are considered for introduction into the NES: VVER, VVER-TOI (modified VVER with increased burnup), VVER-MOX (modified MOX-fueled VVER), MOX- and MNUP-fueled BN-1200 and BREST-1200 (BR-1200) utilizing MNUP fuel (the possibility of starting this reactor on nitride enriched uranium fuel was not considered). It was assumed that the VVER and VVERTOI could be commissioned from the first year of the prognosis period, the BN-1200 from 2030, the BR-1200 and VVER-MOX from 2040. The dynamics of commissioning various reactor units is determined by the optimization problem solution. The schemes of the considered two-component NES models are shown in Fig. 1.

All values used in the calculations are annual average ones, i.e., they correspond to the steady-state reactor operating characteristics, the initial fuel loads and the last SNF discharges were taken into account (in accordance with the data in (Ponomarev-Stepnoy 2016, Adamov 2001, Andrianov et al. 2010)). The BN-1200 is represented in the models separately by the core and the blanket. The costs of NFC services were taken from (Ponomarev-Stepnoy 2016) (average values). Regarding the cost of reactor facilities, a conservative assumption was made, i.e., the overnight capital cost of the BR-1200 is $10 \%$ less, and of the BN-1200 is $10 \%$ more, than that of the VVER, which is $\$ 4,000 / \mathrm{kW}$. The discount rate was assumed to be 5\% (Ponomarev-Stepnoy 2016, Adamov et al. 2017).

The following assumptions were adopted for the growth of NES capacities: $43 \mathrm{GW}$ in 2030, $64 \mathrm{GW}$ in 2050, and $118 \mathrm{GW}$ in 2100 (Adamov et al. 2017). To take into account the boundary effects, the prognosis horizon was extended up to 2150 (150 GW in 2150). The export of reactor technologies and NFC services was not considered. The calculations were carried out taking into account the prehistory of the Russian NES deployment and on the assumption that natural uranium reserves amount to 600 thousand tons, and the capacity of facilities for re-

1 LGC can be considered a system-wide analogous to the levelized unit electricity cost (LUEC) metric.

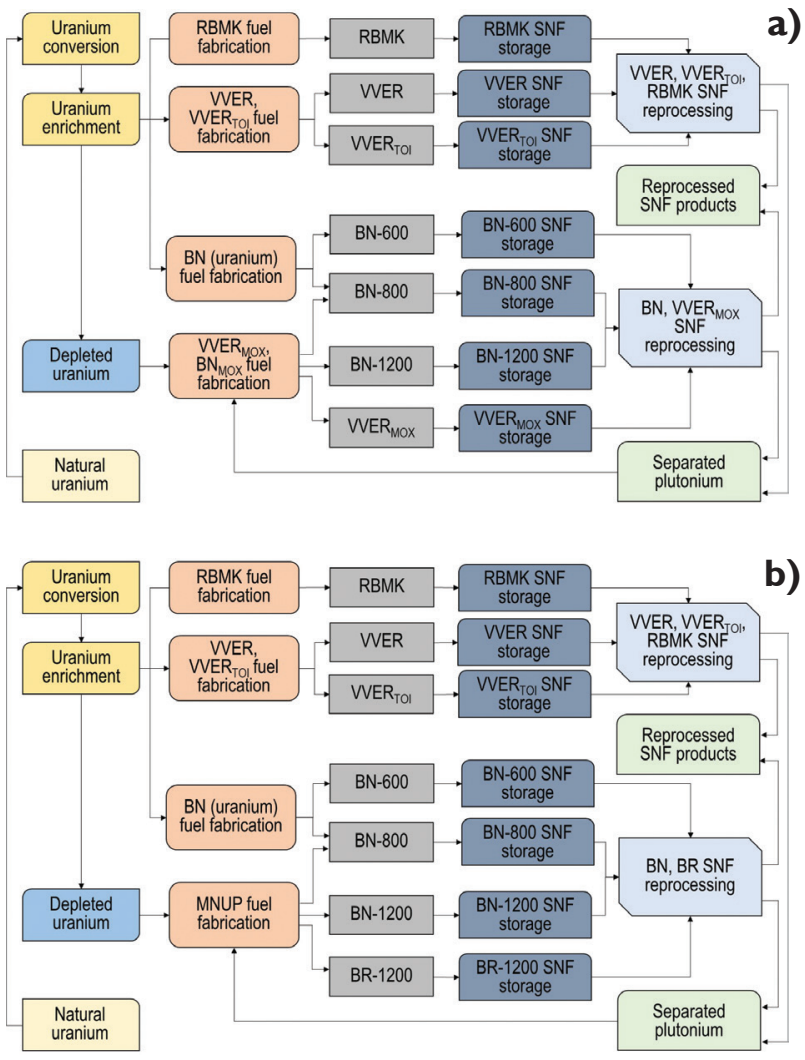

Figure 1. The main structural elements of the two-component NES models: a) Option 1; b) Option 2

processing SNF from all reactor types (including RBMK) will not exceed 2,000 t HM (which approximately corresponds to the total capacities of RT-1,-2, PDC, PDPC). The loading structure of SNF reprocessing facilities is determined by the optimization problem solution. The duration of SNF cooling prior to reprocessing is assumed to be five years for all reactor types. It is assumed that SNF reprocessing will be centralized. Accumulated by 2015, separated plutonium (ex-weapon and reactor-grade) and plutonium contained in SNF are a resource for the fast reactor fuel (the data were taken from (Andrianov et al. 2010)). No other constraints were imposed on the models. The optimization criterion is minimization of the total discounted costs for the entire development program.

The models developed in accordance with the recommendations (NG-T-5.2 2016, Andrianov et al. 2012) were verified by comparison with the results obtained using the original codes, discussed at the IAEA workshops, which suggests that they are correct. The models make it possible to optimize the NES structure by the criterion of minimum total discounted costs, taking into account multiple resource and infrastructural constraints, to estimate the scale of NFC material flows, requirements for NFC services and products as well as economic performance indicators. They can be used as a starting point for developing multi-objective, stochastic and robust optimization models of a two-component NES. 


\section{Some results of applying the optimization models}

The optimal structures of a two-component NES in both variants are determined on the basis of the requirement to minimize the total discounted costs for the entire development program, taking into account resource and infrastructural constraints. The resource constraints are decisive: they determine the range of reactor technologies to be included in the NES structure to ensure the growth in demand for nuclear energy generation. The infrastructural constraints (the decisive factors are the capacities of SNF reprocessing facilities) have a significant impact on the dynamics of commissioning reactor technologies and the structure of their fuel supply, but do not change the set of technologies.

Taking into account the accepted conservative assumptions regarding the cost data, the levelized unit energy costs of thermal reactors turn out to be less than as compared to fast reactors. At the same time, since the reserves of natural uranium are not enough to ensure the given increase in the NES capacities, NES structures require reactors operating on plutonium fuel: in Option 1: the MOX-fueled BN-1200, in Option 2: the MNUP-fueled BN-1200 and BR-1200, the optimal share of which in
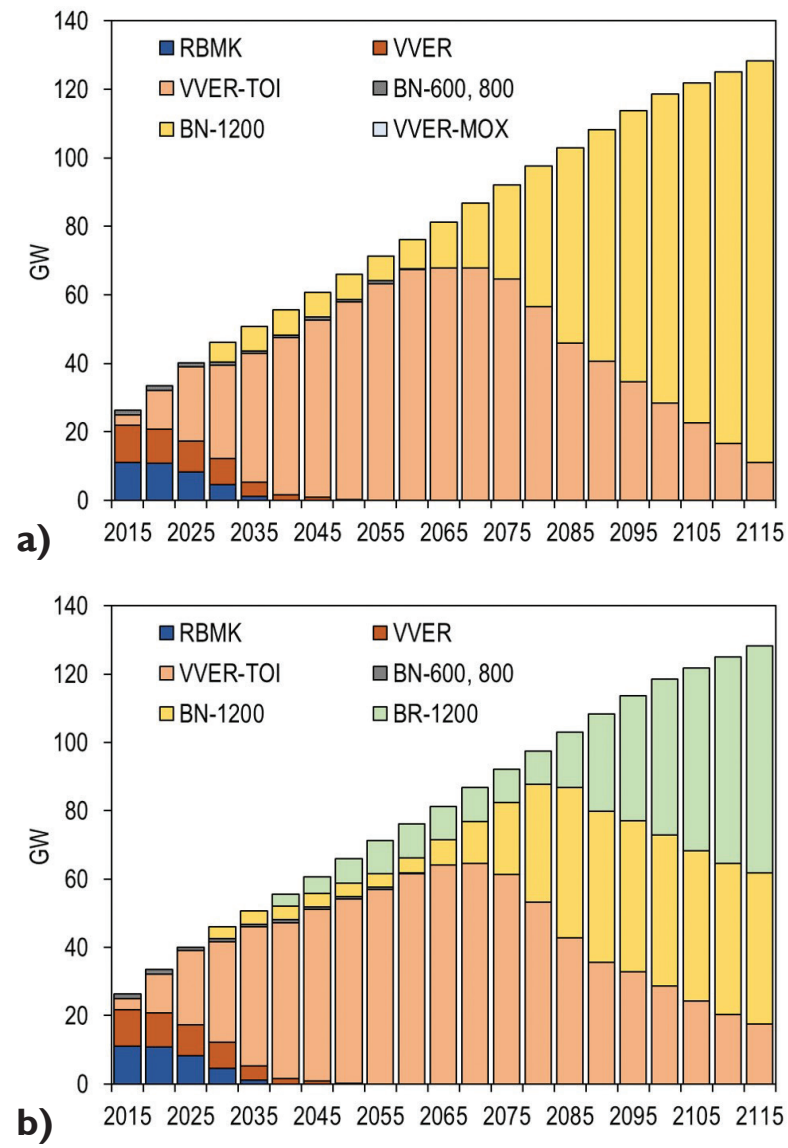

Figure 2. Examples of typical optimal NES structures (annual data are aggregated over five-year periods): a) Option 1; b) Option 2. the system at each time interval is determined by the optimization problem solution. Therefore, the optimal NES structures in the absence of accounting for the infrastructural constraints on the capacities of SNF reprocessing facilities will include the maximum possible (with given uranium reserves) share of thermal reactors (the entire new fleet of thermal reactors to be commissioned after 2015 are the VVER-TOI units in both options). The end of introducing thermal reactors into the system is determined based on the requirement that all commissioned thermal reactors on uranium oxide fuel must be provided with fuel during the entire plant life (natural uranium reserves in this case will be exhausted at the turn of 2120 2130 , the total installed capacities of thermal reactors will not exceed $70 \mathrm{GW}$ ).

This deferred (but more intense in the future) option for introducing fast reactors into the system entails a significant increase in the requirements for SNF reprocessing services in order to timely provide the fast reactors with secondary nuclear fuel to meet the growing demand for nuclear energy generation and compensate for the intensive decommissioning of thermal reactors. This will lead to high peak requirements for SNF reprocessing capacities after 2060, exceeding up to 2.5 times the marginal production capacities of the planned SNF reprocessing facilities, and their subsequent unutilized capacities after the stages of intensive commissioning of fast reactors are completed.

Accounting for the infrastructural constraints on the capacities of SNF reprocessing facilities in accordance with existing plans will lead to their uniform loading and earlier, but less intensive, introduction of fast reactors into the system, which will make it possible to continue operating thermal reactors on uranium oxide fuel after 2115 . The relevant NES structures are shown in Fig. 2.

For the assumptions made in both options, several BN-1200 units with a total installed capacity of about 2.4 GW are introduced into the system in 2030. In Option 1, the total installed capacities of BN-1200 reach 7.2 GW in 2040 and remains at this level until 2060; after which, intensive BN-1200 commissioning begins. In Option 2, in 2040, alongside with the BN-1200 units (4 GW), the BR1200 units are introduced into the system, their installed capacity becomes equal to $9.8 \mathrm{GW}$ in 2056 and remains at this level until 2085. At this time, the BN-1200 units are intensively commissioned; their total installed capacity reaches $44 \mathrm{GW}$ by 2085 and is fixed at this level. Since 2085, the BR-1200 units are actively introduced into the system.

The initial stage of commissioning fast reactors is characterized by the utilization of separated plutonium and mainly by the reprocessing of fast reactor SNF (around 60 and $120 \mathrm{t} \mathrm{HM} / \mathrm{y}$ for Options 1 and 2, respectively), the plutonium from which is used to form new fuel for fast reactors. More intensive commissioning of fast reactors at the initial stage turns out to be economically inexpedient because of the need to deploy the expensive closed NFC infrastructure, the availability of sufficient amounts 
of natural uranium and the possibility to ensure the introduction of fast reactors later on, meeting the restrictions on the closed NFC infrastructure. The stage of intensive fast reactor capacity expansion is characterized by the beginning of large-scale thermal reactor SNF reprocessing.

To ensure the required NES capacity growth within the assumptions made, with uranium reserves of 600 thousand tonnes, the $\mathrm{BN}-1200$ reactors (both MOX- and MNUP-fueled) with a five-year external NFC duration are able to provide the NES with the necessary amount of fuel and capacity growth after the construction of the VVERTOI reactors is stopped and their intensive decommissioning begins. With a five-year external NFC duration, without using the "start on nitride enriched uranium fuel" option, the BR-1200 reactors cannot provide the given growth rate of NES capacities but, in view of the good expected economic performance, this reactor type appears in the system (Option 2) as a BN-1200 companion, which indicates the economic efficiency of such a combination of these fast reactor technologies in the considered conditions. On the contrary, the VVER-MOX reactors are not included under the given assumptions in the optimal NES structure for Option 1. Forcing them into the system (as a strict model constraint) will lead to a change in the NFC material flows, which will be accompanied by an increase in the total discounted cost, thereby making them economically unviable. The share of fast reactors in the NES structure in 2100 reaches $74 \%$ for Option 1 and $75 \%$ for Option 2, respectively (the shares of the $\mathrm{BN}-1200$ and BR-1200 are approximately equal).

The annual natural uranium consumption and requirements for its conversion, enrichment and fuel fabrication for thermal reactors are determined by the program of their commissioning, and the peak for both options falls on the beginning of the 2060s. By 2100 , about $90 \%$ of the available natural uranium for Option 1 and $86 \%$ for Option 2 will be consumed. The remaining part of the natural uranium will be consumed after 2100 to provide fuel for thermal reactors which have not exhausted their plant life by the end of the prognosis interval.

For Options 1 and 2:

- the maximum conversion requirements will be 8.2 and 7.6 thousand $t$ per year;

- the maximum uranium enrichment requirements will be $\sim 7$ and 6.5 million $\mathrm{kg}$ SWU per year;

- the requirements for thermal reactors fuel fabrication will not exceed 1,200 and 1,100 t HM per year.

The average annual requirements for manufacturing MOX (Option 1) and MNUP (Option 2) fast reactor fuels (including the first cores) until their intensive commissioning (approximately, in 2060s) will be $\sim 50$ and $100 \mathrm{t}$ HM per year, respectively. By 2100, these requirements will increase for Options 1 and 2 to about 650 and $950 \mathrm{t}$ HM per year.

The fast reactor fuel fabrication involves the stored separated plutonium (initially) and plutonium extracted during the reprocessing of SNF from thermal and fast reactors (when the separated plutonium is run short). Although the specific SNF reprocessing costs are higher for fast reactors as compared to thermal reactors, less reprocessed SNF of fast reactors is needed to obtain the required amount of plutonium for producing fuel for fast reactors; therefore, it is more economically feasible to reprocess SNF from fast reactors. Thus, if it is enough to extract plutonium from fast reactor SNF to provide new fuel for fast reactors, the reprocessing of SNF from thermal reactors is postponed until an additional amount of plutonium is required. Since the plutonium content in VVER-TOI SNF is higher than that in VVER SNF, and the reprocessing costs are the same, the reprocessing of VVER SNF begins only after all available VVER-TOI $\mathrm{SNF}$ is reprocessed. For the scenario assumptions made, the reprocessing of RBMK SNF is economically inconvenient in both cases. The loading structure of SNF reprocessing facilities is determined by the material balances and economic parameters of the NFC process stages. For Option 1, during the period from 2064 to 2100 , more than $50 \%$ of the SNF reprocessing capacity is involved in reprocessing SNF from thermal reactors; after 2100, most of the SNF reprocessing capacity is involved in reprocessing SNF from fast reactors. For Option 2, during the period from 2045 to 2087, SNF from thermal reactors is mostly reprocessed (more than $50 \%$ of the capacity is involved); after 2087 , SNF from fast reactors is reprocessed. The total capacity of SNF reprocessing facilities will not exceed 2,000 t HM per year for both options (average load is 83 and $88 \%$ for Options 1 and 2, respectively).

The maximum amount of SNF accumulated in the at-reactor and away-from-reactor storage facilities will be about 65 thousand t HM (2064) for Option 1 and 50 thousand t HM (2052) for Option 2, respectively. The amount of SNF will be reduced to 47 and 29 thousand $t$ by 2100 for Options 1 and 2, respectiveely (not all SNF after five-year cooling will undergo reprocessing). Since there is no economic feasibility in reprocessing RBMK SNF, it will remain in the SNF storage facilities in the amount of about 19 thousand t HM for the entire prognosis interval in both options.

In the considered base case of the cost data under the assumptions made, the difference between the options in the total discounted costs for the entire development program is less than $1 \%$. Considering significant uncertainties in the cost data on NFC services and the construction of nuclear power plants, it is of interest to evaluate the impact of these uncertainties on the economic performance of the options (LGC metric) and the spreads in capacities of different reactor types in the optimal NES structures.

The performed calculations of the optimal NES structures (1000 sample scenarios were generated with different cost data from the range of their uncertainties; the spread in economic data was taken from (Ponomarev-Stepnoy 2016), without taking into account the infrastructural con- 

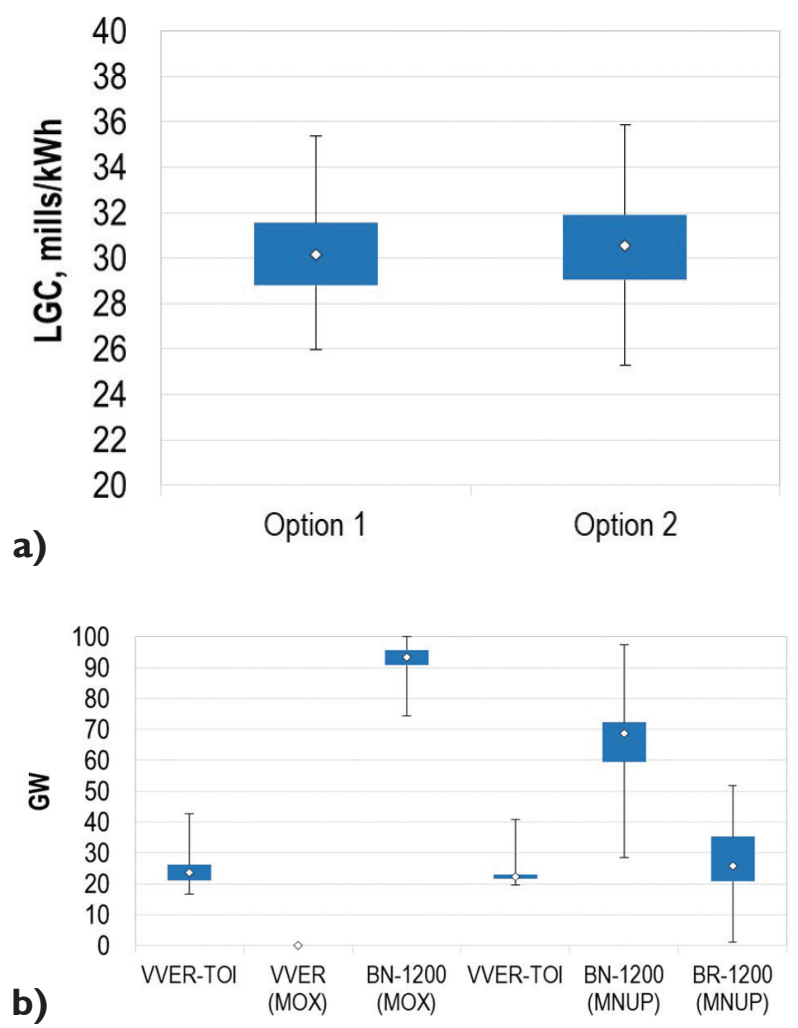

Figure 3. The spread in LGC (a) and total installed capacities of different reactor types in the optimal NES structures in 2100 (b) due to uncertainty in cost data (it is shown maximum and minimum values, 25 and $75 \%$ percentiles, average values)

straints) show that both two-component NES options can be considered statistically indistinguishable by the LGC metric since the $95 \%$ confidence uncertainty intervals are overlapping (Fig. 3a). Consequently, it is impossible to make an unambiguous judgment regarding the prospects of any of the options considered for this economic performance metric, taking into account the existing uncertainties in the cost data. The spread in the total capacities of various reactor types in the relevant optimal NES structures is shown in Fig. 3b. Note that the BN-1200 is always present in the NES structure under the conservative assumptions made, but the most effective companion to it is not the VVER-MOX, but the BR-1200, simultaneous operation with which (subject to the attainability of the expected economic performance) can provide the minimum electricity production cost.

\section{Discussion}

Due to the limited scope of the study, the results of this analysis obviously cannot form the basis for management decisions, but they are quite sufficient to demonstrate the usefulness of using an optimization approach for the purposes of the scenario analysis of national nuclear energy development. Given the assumptions on the costs of NFC process stages and construction of reactor facilities, resource and infrastructural constraints, a gradual moderate transition to a closed NFC turns out to be economically feasible, making it possible to fully master the closed NFC technologies, reduce the existing significant uncertainties in the cost data and make more reasonable judgments regarding the most economically preferred option in the future.

To ensure the given growth rate of NES capacities in the current conditions, there is no need for a fast reactor with high fuel breeding parameters and, as a result, there is no economically determined need to utilize excess plutonium in thermal reactors (other considerations that can make this technological option reasonable are not discussed). The performed evaluations confirm the thesis that, if it is expected to introduce fast reactors in future, the question of using plutonium in thermal reactors requires detailed examination, because there are no irrefutable arguments indicating the feasibility of this option (Muravyev 2014, Andrianov and Schwenk-Ferrero 2017, Gao et al. 2017, Kagramanyan 2018, Ponomarev-Stepnoy and Tsibulsky 2007).

On the contrary, in the long term, the technological diversity of the fast reactor component in the two-component NES structure, suggesting a combination of different types of fast reactors, seems to be economically viable (as indicated in (Andrianov et al. 2012, Andrianov et al. 2016, Andrianov et al. 2017)). In this case, the NES structures are more stable in regard to possible changes in the main technical and economic parameters of the system, which is confirmed by the results of the analysis based on robust and stochastic optimization approaches (Andrianov 2012, Andrianov et al. 2017). This fact confirms the structural stability (robustness) of the found optimal structures of the national NES, which speaks of fast reactors as the basis of large-scale nuclear energy.

\section{Conclusion}

The results of the analysis of the two-component NES structures illustrate the capabilities of the developed optimization models, demonstrating the characteristic structural features of NESs based on fast reactors for conservative model assumptions. Under the accepted limitations and assumptions, the present study determined the types of reactors required for ensuring the sustainable development of the national NES as well as optimal terms and rates of their commissioning. Estimations were made of the required scale of NFC facilities utilization (uranium mining, conversion, enrichment, fuel fabrication, SNF reprocessing, SNF and RW storage facilities). The characteristic features, similarities and differences of both options were also indicated.

The analysis indicated the economic feasibility of technological diversification of the fast reactor component in the two-component NES, which makes it possible to form a robust (stable in regard to possible changes in 
technical, economic and scenario parameters) structure of NES in the future. It has been demonstrated that, given the existing uncertainties in the cost of NFC services and reactor technologies, it is impossible to make a rea- sonable conclusion regarding the greatest attractiveness of any of the options considered in terms of the levelized generation costs.

\section{References}

- Adamov EO (2001) (Ed.) White Book of Nuclear Power Engineering. Moscow: JRC "NIKIET” Publ., 270 pp. [In Russian]

- Adamov EO, Dzhalavyan AV, Lopatkin AV, Molokanov NA, Muravyov EV, Orlov VV, Kalyakin S G, Rachkov VI, Troyanov VM, Avrorin EN, Ivanov VB, Aleksakhin RM (2012) Conceptual framework of a strategy for the development of nuclear power in Russia to 2100 . Atomnaya Energiya [Atomic Energy], 112(6): 391-403. https://doi. org/10.1007/s10512-012-9574-x

- Adamov EO, Mochalov YuS, Muravyev EV, Rachkov VI (2017) Large-scale two-component nuclear power with a closed nuclear fuel cycle on the fast neutron reactors basis. Report to SC "Rosatom". Moscow. SC "Rosatom" Publ., 67 pp. [In Russian]

- Alekseev PN, Asmolov VG, Gagarinsky AYu, Kukharkin NYe, Semchenkov YM, Sidorenko VA, Subbotin SA, Tsibulsky VF, Shtrombakh YI (2011) The strategy for the development of nuclear energy in Russia until 2050. Atomnaya Energiya [Atomic Energy], 111(4): 183-196.

- Alekseev PN, Asmolov VG, Gagarinsky AYu, Kukharkin NYe, Semchenkov YuM, Sidorenko VA, Subbotin SA, Tsibulsky VF, Shtrombakh YaI (2012) On the Russian Nuclear Power Industry Development Strategy until 2050. Proc. of the 8-th International Scientific and Technical Conf. "Safety, Efficiency and Economics of Nuclear Power Industry". Moscow. [In Russian]

- Alekseev PN, Gagarinsky AYu, Kukharkin NYe, Semchenkov YuM, Sidorenko VA, Subbotin SA, Tsibulskii VF, Shimkevich AL, Shtrombakh YI (2017) Strategic view on nuclear power in Russia at the present stage, Atomic Energy [Atomic Energy], 122(3): 123-126. https://doi.org/10.1007/s10512-017-0249-5 [In Russian]

- Andrianov AA (2012) Approaches and software for multi-objective optimization of nuclear power structures. Sustainability, 4: 721-739. https://doi.org/10.3390/su4040721

- Andrianov AA, Korobeinikov VV, Poplavskaya EV, Rachkova EN, Fedorova EV (2010) Optimization studies of the Russia's nuclear power industry structure with thermal and fast neutron reactors by the MESSAGE software. Izvestia vuzov. Yadernaya Energetika [News of Higher Education Institutions. Nuclear Power Engineering], 2: 156-164. [In Russian]

- Andrianov AA, Korovin YuA, Fedorova EV (2012) Optimization of nuclear energy systems by means of the energy planning tool MESSAGE. Obninsk. INPE NRNU MEPhI Publ., 132 p. [In Russian]
- Andrianov AA, Kuptsov IS, Utyanskaya TV (2016) Application of optimization methods for nuclear energy system performance assessment by the MESSAGE software. Izvestiya vuzov. Yadernaya Energetika [News of Higher Education Institutions. Nuclear Power Engineering], 1: 70-80. [In Russian]

- Andrianov AA, Kuptsov IS, Utyanskaya TV (2017) Some results of multicriterial and robust optimization of nuclear power systems structure. Atomnaya Energiya [Atomic Energy], 123(1): 9-14. https://doi.org/10.1007/s10512-017-0293-1

- Andrianov AA, Schwenk-Ferrero A (2017) Comparison and screening of nuclear fuel cycle options in view of sustainable performance and waste management. Sustainability, 9(9:1623): 1-31.

- Gao R, Nam HO, Ko WI, Jang H (2017) National options for a sustainable nuclear energy system: MCDM evaluation using an improved integrated weighting approach. Energies, 10(12), 24 pp.

- Kagramanyan VS (2018) I'm upset for the French. Available at: http://atominfo.ru:17000/hl?url=webds/atominfo.ru/en/news/e0178. $\mathrm{htm} \& \mathrm{mime}=\mathrm{text} / \mathrm{html} \&$ charset=windows- 1252 [Accessed: July 05, 2018)]

- MESSAGE - Model for Energy Supply Strategy Alternatives and their General Environmental Impacts. User manual (DRAFT). International Atomic Energy Agency: Vienna, Austria.

- Messner S, Strubergger M (1995) User's guide for MESSAGE III, WP-95-69, IIASA.

- Muravyev EV (2014) Fuel supply of nuclear power with the fast reactors involving. Izvestiya Rossiyskoy akademii nauk. Energetika [Bulletin of the Russian Academy of Sciences. Power Engineering], 5: 75-86.

- NG-T-5.2 (2016) Modelling nuclear energy systems with MESSAGE: a user's guide, IAEA Nuclear Energy Series No NG-T-5.2. Vienna, 126 pp.

- Ponomarev-Stepnoy NN (2016) (Ed.) Two-Component Nuclear Power System with Thermal and Fast Reactors in a Closed Nuclear Fuel Cycle. Moscow: Tekhnosfera Publ., 160 pp. [In Russian]

- Ponomarev-Stepnoy NN, Tsibulsky VF (2007) Assessment of the effectiveness of mixed uranium-plutonium fuel in VVER. Atomic Energy [Atomic Energy], 103(5): 833-835. https://doi.org/10.1007/ s10512-007-0132-x 